\title{
Healthcare Encounters Verbatim Description
}

National Cancer Institute

\section{Source}

National Cancer Institute. Healthcare Encounters Verbatim Description. NCI Thesaurus.

Code C124023.

The verbatim description of the healthcare encounter being reported. 